$R M x A C, \mathbf{5 3}, 18-22(2021)$

(c) 2021: Instituto de Astronomía, Universidad Nacional Autónoma de México

https://doi.org/10.22201/ia.14052059p.2021.53.05

\title{
NEW IMPROVEMENTS AT TUG-T60 ROBOTIC TELESCOPE
}

\author{
Murat Dindar ${ }^{1}$, Yucel Kilic ${ }^{1}$, Ali Tat ${ }^{1}$, Saniye Dindar ${ }^{1}$, and Ekrem Kandemir ${ }^{1}$
}

RESUMEN

El telescopio robótico T60 de TUBITAK (el Consejo de Investigacón Científica y Tecnológica de Turquía) está controlado formalmente por el software OCAAS de código abierto (TALON). El telescopio se instaló en 2008, pero la modernización y las mejoras son necesarias para aumentar el ciclo útil del telescopio. En este estudio, presentamos los desarrollos y los trabajos de modernización para mejorar la operatividad del T60.

\section{ABSTRACT}

TUBITAK (Scientific and Technological Research Council of Turkey) National Observatory T60 Robotic Telescope is controlled by open source OCAAS software, formally named as TALON. The telescope was installed in 2008. It is still young, but the obsolescence will come for the telescope eventually. Therefore the modernization and improvements are needed to make the telescope lifecycle longer. In this study, we present the developments and modernization works to enhance the T60 maintainability.

Key Words: instrumentation: detectors - telescopes

\section{INTRODUCTION}

The robotic telescope systems have the advantages of operating costs with high efficiency and scientific productivity (Boyd et al. 1985; Drummond et al. 1995). Some demonstrations of a scientific usage of the robotic telescope were given in Akerlof et al. 2003; Boyd et al. 1985; Dindar et al. 2015; Drummond et al. 1995; Ferrero et al. 2010 and Nekola et al. 2010. The term robotic in telescope systems generally stands for guiding a telescope to given position and take images or more complicated tasks (Castro-Tirado 2010).

The term autonomous observatory in astronomy means a robotic telescope and dome are computercontrolled in such manner, that all indispensable actions of observation are done automatically, including the processing of weather conditions, dome driving, choosing objects to observe, exposing by cameras or other optical sensors, taking calibration images, and so forth. Neither human interaction nor activity is necessary for observation (Nekola et al. 2010). Removing humans from the observing process allows faster observation response time, so that makes robotic telescopes respond quickly to alert broadcasts from satellites and begin observing within seconds. This property lets the astronomers to observe transient events (e.g., gamma-ray bursts and other sources) in the sky. That reason is to moti-

\footnotetext{
${ }^{1}$ The Scientific and Technological Research Council of Turkey (TUBITAK) National Observatory Akdeniz University, 07058 Antalya, Turkey (murat.dindar@tubitak.gov.tr).
}

vate us for implementing the new software module to catch the GRB alert in Talon.

TUBITAK National Observatory (TUG) is located at an altitude of $2500 \mathrm{~m}$, on the top of Bakirlitepe mountain in Saklikent region of the city of Antalya which is located on the Mediterranean coast of Turkey; Lat. $36^{\circ} 49^{\prime} 27^{\prime \prime}$ N., Long. $30^{\circ} 20^{\prime} 08^{\prime \prime}$ E. It resides in a longitudinally important region because there is no other active observatory along and around this longitude on which TUG is located. Besides, it fills a significant gap, on the map from far east to the west of Europe.

TUG deserves to point out that the Observatory site is exceptionally good in terms of climatological conditions. The observatory has 1.57 " average seeing value and 210 clear nights/year (Ozisik et al. 2015). Four active telescopes are being operated at the site; the largest being RTT150 followed by a T100 fully automated, T60 and $45 \mathrm{~cm}$ ROTSE III-d robotic telescopes. Two more new robotic telescopes will be in operation soon.

0.60 -meter fully robotic T60 telescope was installed in 2008. The first light was taken in September 2008. The telescope is dedicated to object-based photometric observations. This telescope is mainly used in multi-band photometric observations of variable stars as well as follow-up observations of GAIA or GRB alerts (Table 1).

\section{SYSTEM ARCHITECTURE}

T60 is controlled by Talon software on opensource GNU/Linux platform(Dindar et al. 2015). The telescope can be operated on robotic mode, and 


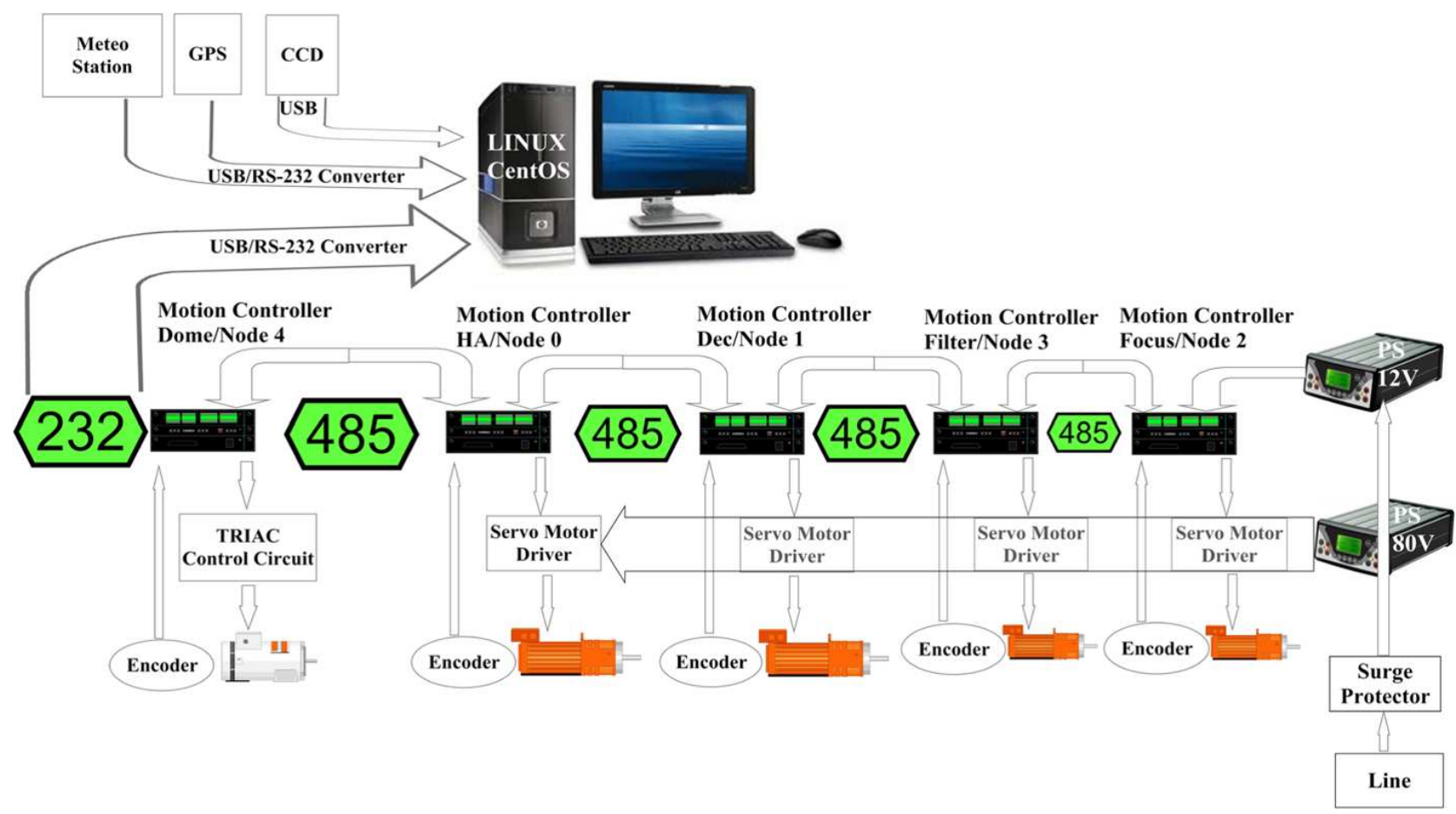

Fig. 1. T60 hardware architecture.

TABLE 1

T60 TECHNICAL SPECIFICATIONS

\begin{tabular}{lc} 
Optical design & Ritchey-Chrétien \\
Main Mirror diameter & $600 \mathrm{~mm}$ \\
Focal Length & $6000 \mathrm{~mm}$ \\
Focal Ratio & $\mathrm{f} / 10$ \\
Resolving Capacity & $0.19 "$ \\
Image Scale & $34 " / \mathrm{mm}$ \\
Dome & Ash-Dome, R \\
& 4.42 dia. \\
\hline
\end{tabular}

the observations are made as object-oriented. The servo motors are used in order to control the axes $\mathrm{RA}$, Dec, focus and filter. The single-phase AC motor is used in order to control the dome. All motors are controlled in Closed Loop by using the motor drivers and the motion controller cards. Every motion controller card is independent and programmed for the distinct processes Figure 1.

T60 telescope control software, Talon, provides the users to control the software, Talon, provides the users to control the telescope both in manual mode (by GUI) and in robotic mode (by batch mode). The users are able to prepare the observations by using the tools of Talon, such as mksch, telsched. The software architecture of Talon is based on the daemon

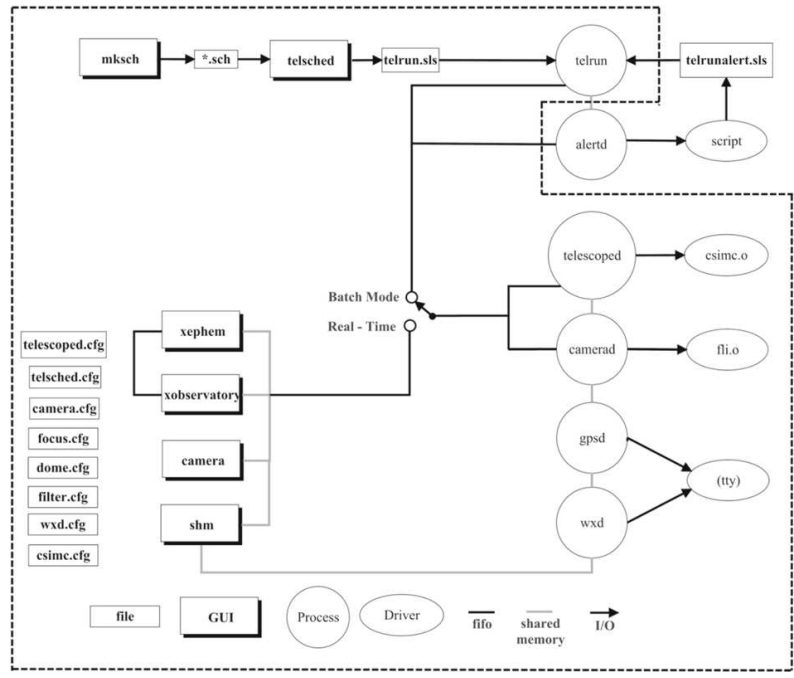

Fig. 2. T60 software architecture.

processes. It has two main daemons of telrun and telescoped. The daemon, telrun operates the schedule on the robotic mode by sending the appropriate command to the daemon, telescoped. The daemon telescoped controls the dome, focus, filter wheel, and the telescope axes modules by sending the low-level commands to the motion controllers in Figure 2. 


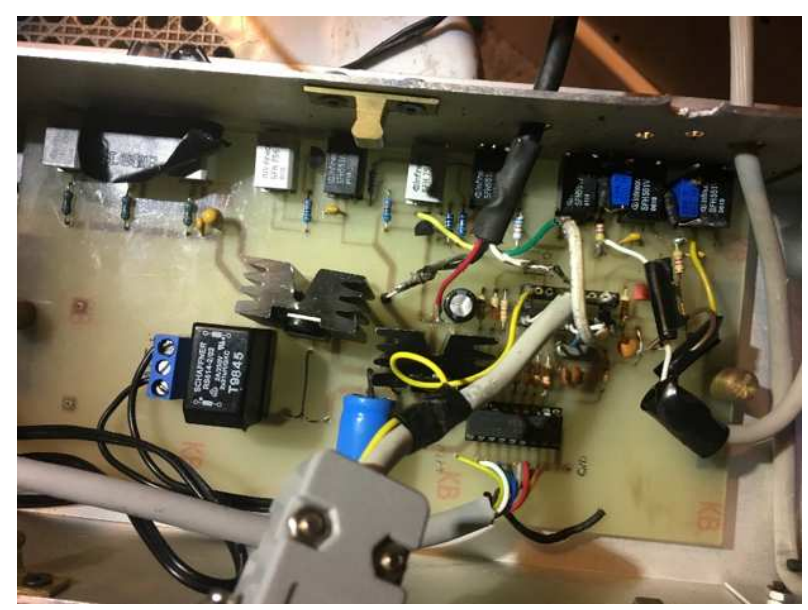

Fig. 3. Dome control system(Previous).

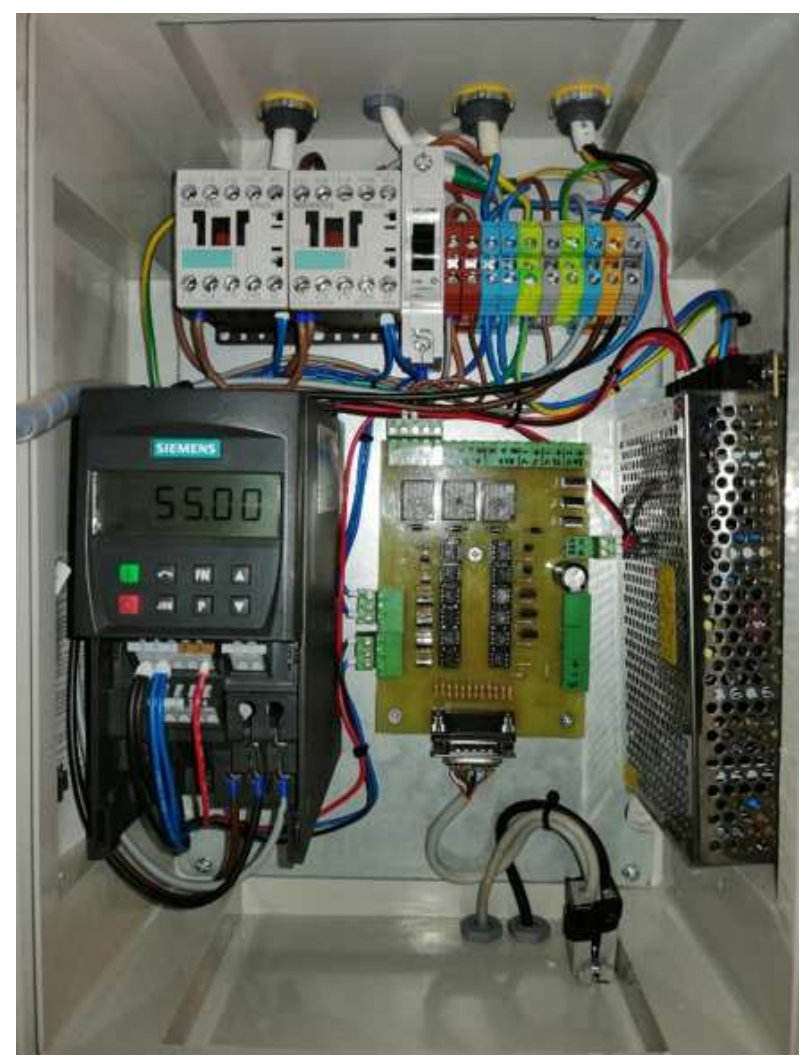

Fig. 4. Dome control system(Upgraded).

\section{DOME CONTROL SYSTEM}

The previous system was custom design an electronic card based on the TRIAC component to control the dome, Figure 3. It had worked well for almost ten years, but the components and connections got complicated after those years. Therefore, it was hard to maintain and find an existing problem.

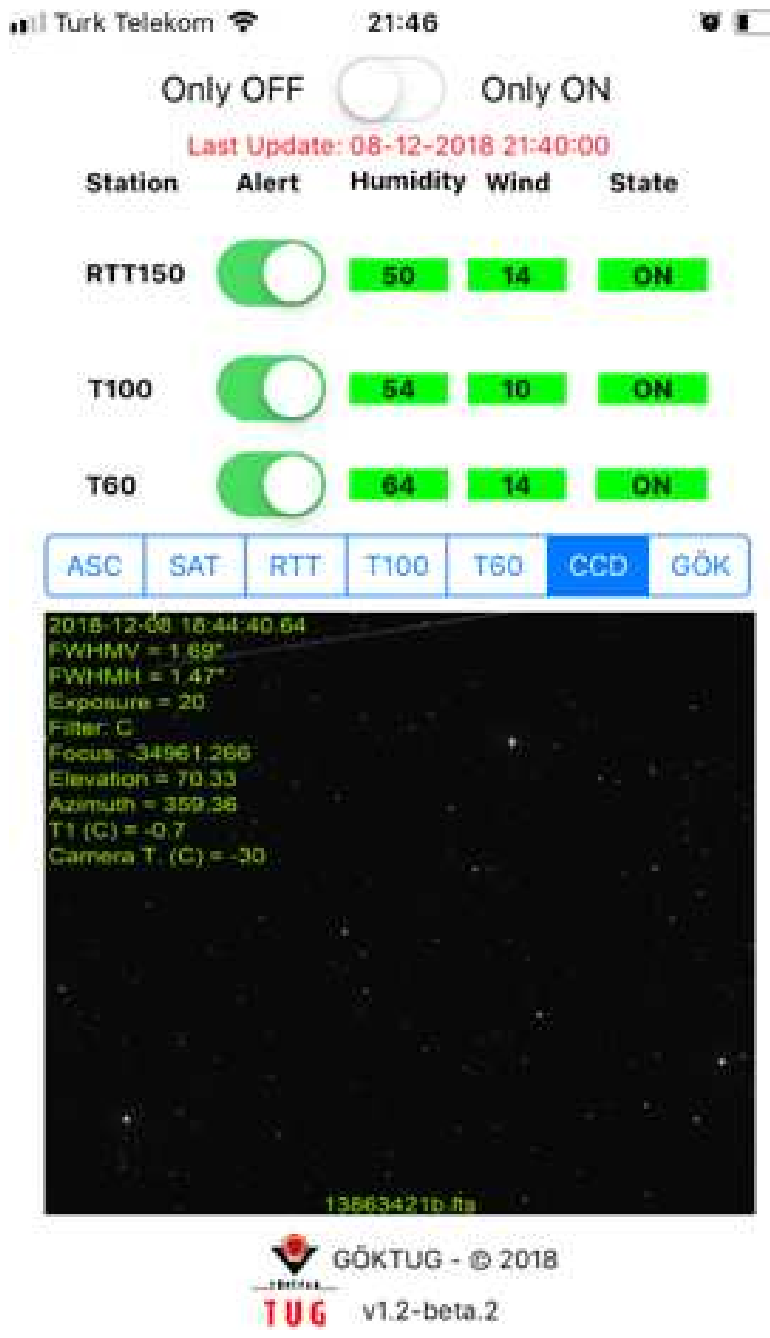

Fig. 5. T60 iOS application, Goktug.

The modernization was needed. For that purpose, it is thought that the new commercial-off-shelf products and custom design cards could be used together to build a more modular system. The new control system is design on two parts Figure 4 .

The first part is the electronic-coupling circuit. It aims that the connection between the controls card, Node Card, of the telescope, and the AC Motor Speed Controller. The second part is the Controller itself. We used the Micromaster-420 as AC Motor Speed Controller. The main advantage of that approach is to adjust the speed of the motor at Dome, so the basic control logic could be applied to the dome movement.

\section{OBSERVATION REPORT SYSTEM}

The report system is designed for two purposes. The first is to summarize the statistical parameters of the telescope, such as weather conditions, obser- 


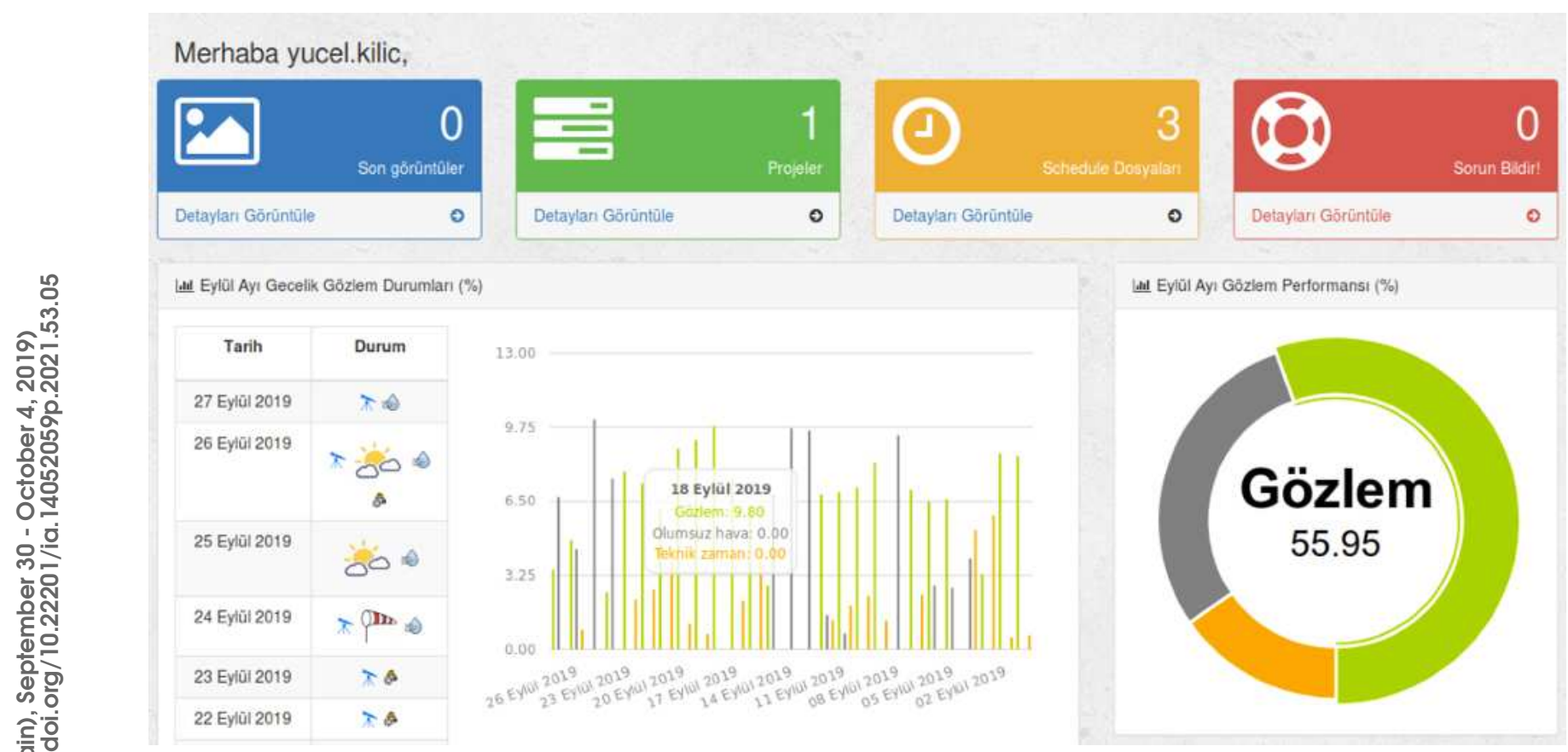

Fig. 6. T60 observatory report system.

vations status, technical downtime, etc. The second is to notify the project investigators to inform them of ongoing observations nightly by sending email and error notifications Figure 6. Thus the investigator will be able to track his/her project easily.

The system is implemented in Python using Django MVT Web Framework. The Django is a high-level Python Web framework that encourages rapid development and clean, pragmatic design. By taking advantage of Django and Python, the modern software packages, astropy, numpy, pandas, etc. written in Python could be easily implemented in a single architecture.

A mobile application called GOKTUG running on the iOS platform was developed to monitor ongoing observations, weather, and technical conditions instantly, as shown in Figure 5. Thus, in the case of a negative situation, the associated staff are informed immediately for the intervention. The Android version of this application is currently being developed.

\section{CCD CAMERA UPGRADE}

The motivation is to not only change the camera with a new one but also upgrade it by taking consideration of the improvements in today's CCD technology. For that purpose, Andor iKon-L 936 (Table 3) has been selected after the market research. The market research was not only the reason to make a
TABLE 2

FLI CAMERA TECHNICAL SPECIFICATIONS

\begin{tabular}{lc}
\hline Model & FLI ProLine 3041-UV CCD \\
Chip & Fairchild CCD 3041-UV \\
Pixel & $2048 \times 2048$ pixel \\
Pixel Size & $15 \times 15 \mu m$ \\
Sensor Sizes & $30.7 \times 30.7 \mathrm{~mm}$ \\
Gain & $1.21 \mathrm{e}^{-} / \mathrm{ADU}$ \\
Noise(RMS) & $10 \mathrm{e}^{-} / \mathrm{pixel} / \mathrm{sec}$ \\
Bias Level & $1730 \mathrm{count}$ \\
Dark Current & $0.06 \mathrm{e}^{-} / \mathrm{pixel}^{-\mathrm{sec}}$ \\
Full Well Capacity & $100,000 \mathrm{e}^{-}$ \\
Digitization & $16 \mathrm{bit}$ \\
Cooling & $\mathrm{TE}+\mathrm{Air}, \mathrm{Scalar} \Delta t-55 \mathrm{C}^{\circ}$ \\
Interface & $\mathrm{USB} 2.0$ \\
Readout Speed & $2.5 \mathrm{sec}$ \\
Pixel Scale & $0.51^{\prime \prime} / \mathrm{pixel}$ \\
FoV & $17.4^{\prime} \mathrm{x} 17.4^{\prime}$ \\
\hline
\end{tabular}

choice, but it also used in other telescopes, RTT150 and had experience on it (Figure 8).

Although Talon control software has already supported different camera models such as SBIG, FLI, Apogee, etc. because of polymorphic methods in Talon camera library, the Andor camera is 


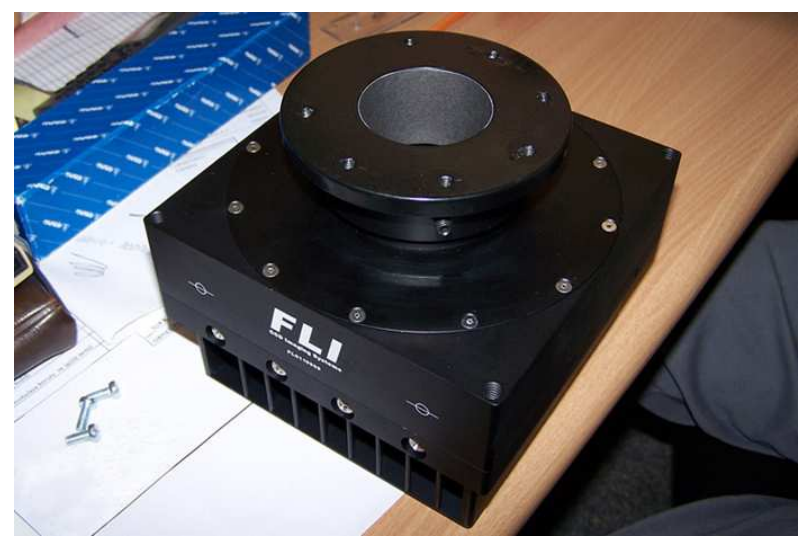

Fig. 7. T60 ccd camera(Previous).

not supported. The main challenge of the integration camera is to implement Andor Linux SDK in Talon. Talon has different modules such as camerad, camera-GUI, autofocus tool, and all modules that need separate connections to the camera driver. But that causes the problems because of having single thread behavior and synchronous SDK methods of Andor Camera. If one module had a connection with the Camera, other modules could not make any connections to Camera. Therefore, all modules which use the SDK methods should be designed and implemented.

The solution is to make a central daemon to handle all low-level camera operations via SDK. The camerad is chosen to implement the central camera daemon, and all other sub-modules communicate with camerad to handle camera operations.

\section{CONCLUSION}

Talon, Observatory Control Software (OCS) has been improving at TUG-T60 Robotic Telescope. The modular structure of Talon helps us to make improvements. New upgrades on the hardware architecture of the telescope will be planned and scheduled soon.

\section{REFERENCES}

Boyd, L. J., Genet, R. M., \& Hall, D. S. 1985, ST, 70, 16 Castro-Tirado, A. J. 2010, AdAst, 2010, 60

Dindar, M., Helhel, S., Esenoğlu, H., \& Parmaksizoğlu, M. 2015, ExA, 39, 21

Drummond, M., Bresina, J., Edgington, W., et al. 1995, PASP, 70, 112

Ferrero, A., Hanlon, L., Felletti, R., et al. 2010, AdAst, 2010,36

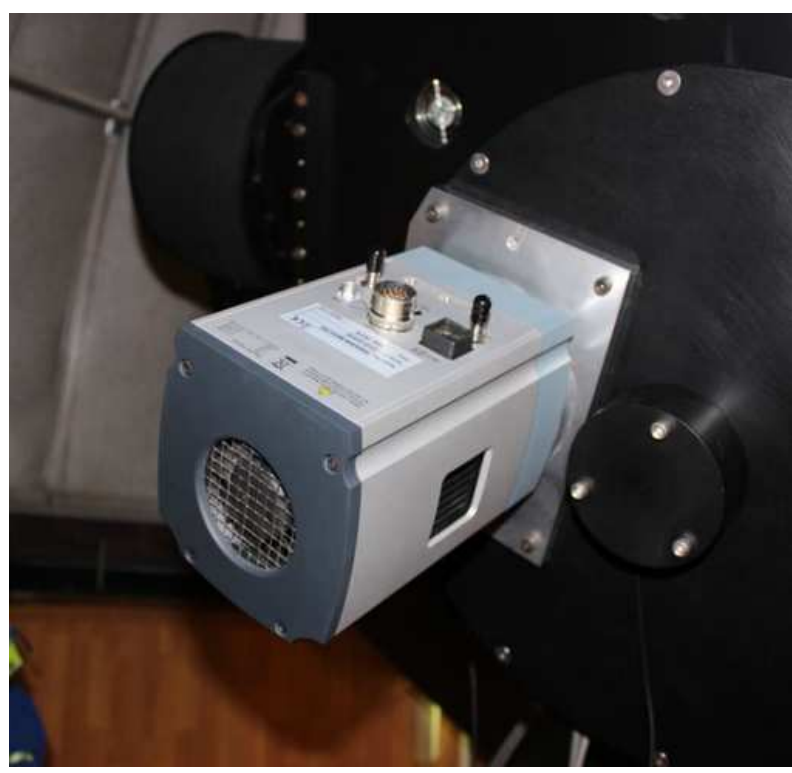

Fig. 8. T60 ccd camera (Upgraded).

TABLE 3

ANDOR CAMERA TECHNICAL SPECIFICATIONS

\begin{tabular}{lc}
\hline Model & Andor iKon-L 936 BEX2-DD \\
Chip & e2v CCD42-40 \\
Pixel & $2048 \times 2048$ pixel \\
Pixel Size & $13.5 \times 13.5 \mu m$ \\
Sensor Sizes & $26.6 \times 27.6 \mathrm{~mm}$ \\
Gain & $1.1 \mathrm{e}^{-} / \mathrm{ADU}$ \\
Noise(RMS & $6.9 \mathrm{e}^{-} / \mathrm{pixel} / \mathrm{sn}$ \\
Bias Level & $1959 \mathrm{count}$ \\
Dark Current & $0.004 \mathrm{e}^{-} / \mathrm{pixel}^{-\mathrm{sn}}$ \\
Full Well Capacity & $100,000 \mathrm{e}^{-}$ \\
CCD Modes & High Sensivity-High Capacity \\
Digitization & $16 \mathrm{bit}$ \\
Cooling & $\mathrm{TE}+$ Air, Scalar $\Delta t-60 \mathrm{C}^{\circ}$ \\
Interface & $\mathrm{USB}^{\circ} 2.0$ \\
Readout Speed & $5 / 3 / 1 / 0.005 \mathrm{Mhz}$ \\
Pixel Scale & $0.456^{\prime \prime} / \mathrm{pixel}^{\prime}$ \\
FoV & $15.6^{\prime} \mathrm{x} 15.6^{\prime}$ \\
\hline
\end{tabular}

Nekola, M., Hudec, R., Jelínek, M., et al. 2010, AdAst, 2010, 44

Ozisik, T. \& Ak, T. 2004, A\&A, 422, 1129 